\title{
Telemedicina como Instrumento de Integração entre Pacientes e Médicos
}

\author{
Telemedicine as an Instrument for Integration between Patients and Doctors \\ Telemedicina como Instrumento de Integración entre Pacientes y Doctores
}

Recebido: 20/07/2021 | Revisado: 22/07/2021 | Aceito: 22/07/2021 | Publicado: 23/07/2021

\author{
Cáledi Ataíde \\ ORCID: https://orcid.org/0000-0002-6561-6100 \\ Centro Universitário da Fundação Assis Gurgacz, Brasil \\ E-mail: calediataide@hotmail.com \\ Odirlei Antônio Magnagnagno \\ ORCID: https://orcid.org/0000-0003-0500-2251 \\ Centro Universitário da Fundação Assis Gurgacz, Brasil \\ E-mail: odirlei@fag.edu.br
}

\begin{abstract}
Resumo
A telemedicina é caracterizada pela aplicação de tecnologia da informação e comunicação nos sistemas de saúde, em especial, quando a distância é um fator elementar. Nos países desenvolvidos, a utilização desse programa de telessaúde é uma realidade, sendo utilizada para diagnóstico, promoção e tratamento de patologias. Esse modelo de prestação de serviço médico coaduna-se com a realidade virtual e com as necessidades da sociedade, uma vez que barreiras geográficas, as quais poderiam impossibilitar procedimentos em saúde, não são um fator de impedimento tão preponderante. O objetivo deste artigo é examinar a opinião dos pacientes acerca da telemedicina e, dessa forma, compreender se utilizariam a telemedicina como serviço de saúde. Este trabalho é uma pesquisa qualitativa exploratória, com coleta de dados por meio de entrevistas semiestruturadas, com 11 pacientes, formuladas pelos pesquisadores contendo 6 perguntas, que foram gravadas e posteriormente transcritas, acerca de suas posições sobre a definição de telemedicina, vantagens/desvantagens, modos de utilização da telemedicina, relação médico-paciente virtual, entre outros aspectos. Como resultado, nota-se que a maioria dos pacientes usaria serviços de telemedicina como a teleconsulta, contudo, os entrevistados ponderaram que priorizam o atendimento presencial, e somente utilizariam essa alternativa tecnológica para acompanhamento clínico ou se inviabilidade da consulta física tradicional.
\end{abstract}

Palavras-chave: Telemedicina; Inteligência artificial; Segurança; Qualidade; Assistência em saúde.

\begin{abstract}
Telemedicine is characterized by the application of information and communication technology in health systems, especially when distance is an elementary factor. In developed countries, the use of telehealth program is a reality, being used for diagnosis, promotion and treatment of pathologies. This model of providing medical services is consistent with virtual reality and the needs of society, since geographical barriers, which could make health procedures impossible, are not such a major impediment. The purpose of this article is to examine patients' opinions about telemedicine and, thus, understand what they know and how they see the applicability of telemedicine in health services. This work is an exploratory qualitative research, with data collection through semi-structured interviews, with 11 patients, formulated by the researchers containing 6 questions, which were recorded and later transcribed, about their positions regarding the definition, advantages/disadvantages, ways of using telemedicine, virtual doctor-patient relationship, among other aspects. As a result, it is noted that the majority of patients interviewed would use telemedicine services such as teleconsultation, however, respondents consider that they prioritize face-to-face care, and would only use this technological alternative for clinical monitoring or if traditional physical consultation is not viable.
\end{abstract}

Keywords: Telemedicine; Artificial intelligence; Safety; Quality; Health care.

\section{Resumen}

La telemedicina se caracteriza por la aplicación de las tecnologías de la información y la comunicación en los sistemas de salud, especialmente cuando la distancia es un factor elemental. En los países desarrollados, el uso de este programa de telesalud es una realidad, siendo utilizada para el diagnóstico, promoción y tratamiento de patologías. Este modelo de prestación de servicio médico está en consonancia con la realidad virtual y con las necesidades de la sociedad, ya que las barreras geográficas, que podrían hacer impedimento para los procedimientos, no constituyen un impedimento tan preponderante. El propósito de este artículo es examinar la opinión de los pacientes sobre la telemedicina y, de manera, comprender si utilizarían la telemedicina como servicio de salud. Este trabajo es una investigación cualitativa exploratoria, con recolección de datos por a través de entrevistas semiestructuradas, con 11 pacientes, formuladas por los investigadores conteniendo 6 preguntas, las cuales fueron grabadas y posteriormente transcritas, sobre sus posiciones sobre definición de telemedicina, ventajas/desvantajas, modos de uso de la telemedicina, relación virtual médicopaciente, entre otros aspectos. Como resultado, se observa que la mayoría de los pacientes utilizarían servicios de 
telemedicina como la teleconsulta, sin embargo, los entrevistados pensaron que priorizar la atención presencial, y solo utilizaría esta alternativa tecnológica para el seguimiento clínico o si la consulta física tradicional es inviable.

Palabras clave: Telemedicina; Inteligencia artificial; Seguridad; Calidad; Cuidado en salud.

\section{Introdução}

Nos centros de saúde onde há aplicação de novas tecnologias da comunicação e informação, ocorre agilidade na execução dos procedimentos médicos. Isso ocorre porque a aplicação da telemedicina é utilizada desde a informatização dos prontuários dos pacientes em rede até atendimento clínico dos doentes. Contudo, a telemedicina como instrumento de prática médica já está presente há algumas décadas, afinal, os médicos já têm utilizado os recursos de telecomunicação, como o telefone e o antigo fax, para ajudarem os seus pacientes (Wen, 2015).

Esta modalidade de atendimento a distância é uma das características que tornam a telemedicina tão aplicável às necessidades atuais do sistema de saúde, haja vista que permite atendimento de pacientes a longa distância, reduzindo o tempo de espera pelo atendimento, bem como a lotação física dos espaços de saúde. Além disso, a telemedicina baseia-se em especialidades que de alguma forma funcionam a partir da solicitação de imagens com a finalidade de identificar certas patologias, como telerradiologia, telecardiologia, teleginecologia, telepatologia, telepediatria, telepneumologia, teledermatologia e telecardiologia pediátrica, que facilitam a teleconsulta através de videoconferência (Morgado, 2015).

Com a revolução técnico-científico-informacional em vigor desde a segunda metade do século XX, todos os sistemas de produção e de serviço no mundo foram sendo alterados, incorporando novas tecnologias. Nesse contexto de inovação, também foram utilizados componentes do sistema de informação, que constitui a telemedicina, a qual ocorre através da utilização de metodologias interativas de comunicação audiovisual e de dados, com o objetivo de assistência, educação e pesquisa em saúde (CFM, 2002).

Por ser um conjunto de conceitos bastante heterogêneo e recente, são diversas as definições para telemedicina. Segundo a Organização Mundial de Saúde como, a telemedicina é "a oferta de serviços ligados aos cuidados com a saúde, nos casos em que a distância é um fator crítico" (Silveira, 2020). Este elemento crítico permite com que conteúdos de informação, coletados por profissionais da área da saúde, possam ser compartilhados entre demais profissionais, o que possibilita discussão de diagnóstico, prevenção e tratamento de doenças com o objetivo de melhorar a saúde da comunidade (Urtiga, Louzada, \& Lúcia, 2004). Assim, a aplicabilidade da telemedicina é bastante ampla nos sistemas de saúde. Dentre as modalidades, existem a teleconsulta, telemonitoramento, teleconsultoria e até mesmo a telecirurgia. Essa diversidade de utilização na prática médica, com compartilhamento de informação clínica dos pacientes, permite com que seja alcançado o objetivo maior da telemedicina que é o de permitir acesso à saúde e, junto a isso, garantir a redução de custos e de tempo e o aumento da qualidade do atendimento (Oliveira \& Chao, 2000).

A teleconsulta é uma das formas mais prevalentes da telemedicina. Essa modalidade permite com que o paciente seja atendido indiretamente por um médico, ou seja, o problema de saúde pode ser tratado à distância, por meio de coleta de informações online e posterior transferência de dados de exames radiológicos para uma plataforma digital, permitindo o profissional concluir o diagnóstico. No que diz respeito à teleconsulta, ela pode ocorrer por serviços seguros de telefonia, videoconferência, chat, e-mail, mensagens instantâneas e aplicativos para dispositivos móveis, que corresponde a uma nova fronteira de interação entre médicos e pacientes (Schmitz et al., 2017).

Outra aplicação da telemedicina ocorre pela telecirurgia. Esta é feita por meio de robôs guiados, a distância, por cirurgiões, os quais têm acesso minuncioso do campo cirúrgico por meio de equipamentos de alta resolução, sem que isso atrapalhe a precisão e a recuperação do paciente. Tais recursos utilizados na telecirurgia também podem servir como realidade virtual e serem usados em treinamento e simulações tridimensionais de casos clínicos, auxiliando na formação técnica de profissionais (Rocha de Oliveira \& Lung Wen, 2000). 
Em países desenvolvidos, como nos Estados Unidos e Canadá, a realidade da teleconsulta, por exemplo, nos serviços hospitalares é mais efetiva, ampliando o acesso à saúde para pacientes de áreas remotas e rurais (Schmitz et al., 2017). Já no Brasil, a teleconsulta ainda não está regulamentada pelo CFM, pois é vedado ao médico a prescrição de tratamento ou outros procedimentos sem exame direto do paciente, salvo casos de urgência/emergência, mas com atendimento presencial subsequente obrigatório (CFM, 2019).

O governo federal, por meio do Ministério da Saúde, já tem utilizado a telemedicina como política pública, por meio do Programa Nacional Telessaúde Redes. Através desse projeto, ocorreu curso de qualificação de equipes em saúde da família, do sistema de atendimento primário do SUS, em 100 unidades básicas de saúde (Maldonado, Cruz, e Marques, 2016). No estado do Rio Grande do Sul, a Secretaria de Saúde do Estado criou o "Respira Net", um serviço de telediagnóstico para doenças do aparelho respiratório, em que são feitos exames de espirometria nos pacientes das UBS, permitindo acompanhamento dos pacientes de doenças respiratórias crônicas (SES/RS, 2014).

Como a norma de 2019 do CFM ainda define que "teleconsulta subentende como premissa obrigatória o prévio estabelecimento de uma relação presencial entre médico e paciente", cabe aos gestores de saúde do país encontrar uma resolução que consiga associar essa norma à telessaúde. Como possibilidade, após um contato pessoal do médico com o paciente, a sequência do tratamento possa ser feita à distância com mais segurança (Jardim, 2019). Logo, um fator importante é a aceitação do paciente em optar por um serviço que envolva a telemedicina, de modo a se sentir confiante na conduta terapêutica que lhe será dada. Talvez, este seja um elemento de resistência, afinal, o processo comunicativo permeia todas as relações interpessoais e a forma como é desenvolvido pode facilitar ou dificultar a confiança e o vínculo do paciente e família com os profissionais de saúde, assim como o vínculo destes com o serviço de saúde, influenciando na adesão ao tratamento (Otani \& Aparecida, 2013).

Devido à pandemia da Covid-19, o Ministério da Saúde publicou a Portaria n. 467/2020, a qual determina, em caráter excepcional e temporário, sobre as ações de telemedicina, com o objetivo de regulamentar e operacionalizar as medidas de enfrentamento da emergência de saúde pública (Ministério da Saúde, 2020). Nessa Portaria, as ações de telemedicina podem ser praticadas tanto no âmbito do Sistema Único de Saúde (SUS), quanto no setor privado, e podem contemplar o atendimento préclínico, de suporte assistencial, de consulta, monitoramento e diagnóstico, desde que o atendimento realizado entre médico e paciente seja feito através de tecnologia que garanta a integridade, a segurança e o sigilo das informações coletadas durante a prática da telemedicina. Assim, devido a essa situação de calamidade de saúde pública, a norma do CFM de 2019 que regulariza telemedicina apenas como acompanhamento após uma primeira consulta presencial, temporariamente enquanto durar a pandemia, não é exigida aos médicos

Assim, este estudo objetiva examinar a opinião dos pacientes acerca da telemedicina e, dessa forma, compreender se utilizariam a telemedicina como serviço de saúde. Com isso, o estudo da pesquisa visa contribuir com a comunidade científica sobre a telemedicina, tendo como base a visão dos pacientes sobre essa realidade, corroborando para a disseminação dos conceitos da telessaúde, além de auxiliar na formatação de estratégias dos grupos de saúde de como melhor utilizar as tecnologias da comunicação e informação para um atendimento mais efetivo que se adeque à realidade do paciente.

\section{Metodologia}

A pesquisa teve a participação de 11 pacientes e ocorreu durante o mês de novembro de 2020 e análise das respostas ocorreu durante o mês de dezembro de 2020, período no qual os pesquisadores realizaram comparações entre as respostas dos pacientes. A pesquisa não apresentou distinção de área de atuação, de gêneros e faixa etária. Os pacientes foram abordados aleatoriamente pelos entrevistadores nos ambulatórios, enfermaria e sala de espera do Hospital São Lucas, sendo eles tanto do SUS quanto do convênio/particular (não SUS). Posteriormente, os dados obtidos foram transcritos no Word, onde foram analisados qualitativamente. Como base metodológica utilizou-se o trabalho de Pereira et al (2018). 
O roteiro que guiou o pesquisador para entrevista com os pacientes era formado por 6 perguntas (Tabela 2). As características de cada entrevistado constam na Tabela 1. Em que a letra "E" significa entrevistado, "ESC" ensino superior completo, "EMC" ensino médio completo, "NS" não SUS e "S" SUS.

Tabela 1: Lista dos Entrevistados.

\begin{tabular}{|c|c|c|c|c|c|c|c|c|c|c|c|}
\hline & E1 & E2 & E3 & E4 & E5 & E6 & E7 & E8 & E9 & E10 & E11 \\
\hline Idade & $25-35$ & $25-35$ & $35-45$ & $46-55$ & $35-45$ & $46-55$ & $25-35$ & $46-55$ & $35-45$ & $46-55$ & $25-35$ \\
\hline Escolaridade & ESC & ESC & ESC & EMC & ESC & EMC & EMC & ESC & ESC & EMC & ESC \\
\hline $\begin{array}{l}\text { Programa de } \\
\text { saúde }\end{array}$ & NS & NS & NS & $S$ & NS & S & S & NS & S & $S$ & NS \\
\hline
\end{tabular}

E: Entrevistado; ESC: Ensino Superior Completo; EMC: Ensino Médio Completo; NS: Não SUS; S: SUS; Fonte: Autores (2021)

Tabela 2: Descrição dos pontos a esclarecer, explicação e referências das perguntas que foram questionadas aos pacientes.

\begin{tabular}{llll}
\hline Pergunta & \multicolumn{1}{c}{ Pontos a esclarecer } & \multicolumn{1}{c}{ Explicação } & Referência \\
\hline 1) Você já ouviu & -Verificar se o paciente sabe da & Telemedicina, em sentido amplo, pode ser definida & \\
falar/sabe & telemedicina. Caso & como o uso das tecnologias de informação e & \\
$\begin{array}{l}\text { sobre } \\
\text { telemedicina? }\end{array}$ & não saiba, ler a definição da & comunicação na saúde, viabilizando a oferta de & Maldonado, \\
& explicação. & serviços ligados aos cuidados com a saúde & Marques, \& Cruz, \\
& & (ampliação da atenção e da cobertura), & 2016
\end{tabular}

2) Você acha que a telemedicina traz alguma vantagem para o paciente?

De quais formas você utilizaria os serviços telemedicina?

Você acredita que

haja

alguma restrição tecnológica para realização de uma teleconsulta?
-Esclarecer as vantagens, se houver, bem como as desvantagens na visão do paciente.

-Tentar trazer exemplos de vantagens e desvantagens da telemedicina.
-Vantagem: É possível economizar seu dinheiro em transporte público, estacionamento e assim por diante, quando você visita seu médico em seu computador ou dispositivo móvel. Além disso, nenhum tempo será desperdiçado em um engarrafamento, o que pode atrasar a consulta com o médico.

Ciommo, 2019

-Desvantagens: A telemedicina facilita que os pacientes mudem continuamente seus médicos, o que resulta em uma redução na continuidade do atendimento. Além disso, esses médicos aleatórios não serão capazes de obter acesso a todas as origens históricas de seus pacientes, resultando em tratamento errático em longo prazo

O novo coronavírus trouxe muita ansiedade por informação. A telemedicina ajuda que o paciente esclareça suas dúvidas. Normalmente, ele procuraria um pronto-socorro. Pelo aplicativo, ele pode receber atendimento médico, da casa dele, por uma equipe muito preparada

Embora tenha havido muitos programas inovadores recentemente, a telemedicina ainda continua sendo tecnologicamente limitada. É possível que as conexões de banda larga funcionem incorretamente, dispositivos de bate papo com vídeo funcionem incorretamente e assim por diante.
Varella, 2020

Ciommo, 2019 


\section{Resultados e Discussão}

A primeira pergunta do questionário tratava-se sobre se o paciente sabe o que se significa/já tinha ouvido algo sobre a telemedicina. Essa pergunta foi realizada no intuito de averiguar o grau de compreensão dos pacientes a respeito da telemedicina, de modo que, quando não sabiam do que se tratava, o entrevistador lia a definição conforme está descrito no questionário. Foram obtidas respostas bastante variadas como "Tipo de atendimento com tecnologia para prestar serviços na medicina" de acordo com o E3; "Acredito que seja atendimento através da internet" segundo o E9 ou ainda "Acho que é atendimento online com o médico" pelo E1. Dessa forma, os entrevistados compreendem a aplicação da telemedicina praticamente para o atendimento médico em forma de consulta, portanto, a teleconsulta. No que diz respeito à essa modalidade de atendimento, ela pode ocorrer por serviços seguros de telefonia, videoconferência, chat, e-mail, mensagens instantâneas e aplicativos para dispositivos móveis, que corresponde a uma nova fronteira de interação entre médicos e pacientes (Schmitz et al., 2017).

Entretanto, a telemedicina engloba elementos bem mais diversificados que a própria teleconsulta. Isso porque a telemedicina também ocorre através da utilização de metodologias interativas de comunicação audiovisual e de dados, com o objetivo não somente de assistência, mas também de educação e pesquisa em saúde (CFM, 2002). Todavia, essas informações podem ser novas para uma grande maioria dos pacientes, tanto é que 9 participantes não souberam definir ou nunca tinham ouvido o termo telemedicina, sendo válido ressaltar que, dos que compreendiam telemedicina, todos eram de convênio/particular e com ensino superior completo e perfil jovem (até 45 anos).

A segunda questão da entrevista solicitava aos pacientes exemplificar vantagens e desvantagens da telemedicina. Para esta pergunta, 5 pacientes responderam como desvantagem a distância física do médico, o que, para eles, poderia comprometer o exame físico e, consequentemente, o diagnóstico do problema que as levaram consultar. Como resposta dessa desvantagem, foram obtidas algumas respostas como "Exame físico fica comprometido, para uma primeira consulta tem que ter um exame no paciente, tocar onde dói" (E1); "O fato do médico não ver o paciente diretamente é ruim, de não pegar no paciente" (E5); "Seria mais difícil pra examinar e ver o que o paciente realmente tem" (E7) e "Acho complicado pra dar um diagnóstico sem examinar um paciente" (E11). Ainda segundo o E3, o distanciamento físico do profissional médico pode comprometer a qualidade da consulta pois "Sem o olho no olho do médico com o paciente acho que fica mais impessoal".

De todos estes que descreveram desvantagens, 5 eram convênio/particular e apenas 1 SUS. Os demais não souberam exemplificar bem as desvantagens, enquanto 2 pacientes SUS (E10, E8) acreditam não haver desvantagens na telemedicina. No quesito vantagem, a principal apontada pelos entrevistados é quanto a não necessidade de deslocamento para a clínica/hospital para ser realizada uma consulta (E1, E2, E3, E6, E7, E8, E10, E11). Esta vantagem apontada é comum para os entrevistados tanto do SUS quanto do convênio/particular. Logo, essas respostas coadunam com o objetivo maior da telemedicina que é o de permitir acesso à saúde e, junto a isso, garantir a redução de tempo ao paciente (Oliveira \& Chao, 2000).

Um ponto importante lembrado pelo E8 é a vantagem da telemedicina no âmbito da Pandemia de Covid-19, em que pacientes com alguma comorbidade podem utilizar uma teleconsulta sem correr risco de se expor ao vírus indo a uma clínica/hospital e assim se prevenir. Para o E1, todavia, mesmo com a possibilidade de poupar tempo quanto ao deslocamento de ir ao hospital, a vantagem da teleconsulta somente ocorreria se fosse para um retorno ou para tirar dúvidas, de modo que a primeira consulta deve ser presencial. Uma vantagem relevante foi descrita pelo E7, quanto à aplicabilidade da telemedicina nos serviços de atenção básica: "Consulta do posto demora muito e se fosse online, que não precisa ser presencial, talvez o médico pudesse atender mais pacientes". Assim, a telemedicina na atenção primária tem o potencial de ampliar as ações dos profissionais de saúde, integrando-os aos serviços de saúde localizados em hospitais e centros de referência e promover prevenção, diagnóstico e tratamento dos pacientes. Nesse sentido, o aspecto primordial da telemedicina é o seu potencial de democratizar o acesso aos serviços de saúde (Maldonado, Cruz e Marques, 2016). 
A terceira questão buscava saber dos pacientes se eles utilizariam alguma forma de atendimento por telemedicina. A maior parte dos pacientes, tanto SUS quanto convênio/particular, afirmaram que usariam sim, entretanto, com ressalvas. De acordo com o E1 "Um retorno de consulta eu usaria a telemedicina, mas a teleconsulta numa primeira vez com o médico eu não usaria" e com E9 "Se fosse um retorno e tendo já a primeira consulta presencial, eu usaria tranquilo". Esses pacientes consideram utilizar a telemedicina como uma ferramenta de acompanhamento/retorno dos seus casos clínicos, considerando importante a primeira consulta acontecer de forma presencial. Dessa forma, após um contato pessoal do médico com o paciente, a sequência do tratamento pode ser feita à distância com mais segurança (Jardim, 2019). Somente 1 paciente afirmou que não utilizaria telemedicina "Não porque prefiro ir no consultório mesmo" (E2).

A próxima questão perguntava aos pacientes se há alguma restrição tecnológica para a prática da telemedicina. A maioria deles ( 7 entrevistados) respondeu que sim, evidenciando opiniões como "Falta de acesso por algumas pessoas prejudica sim usar a telemedicina" (E2), "Acesso à tecnologia não é alcançável a todo mundo" (E11) e "Acho que pra quem tem dificuldade de mexer no celular como eu que sou mais de idade, teria mais problema" (E10). Segundo a pesquisa TIC Domicílios 2019, realizada pelo Comitê Gestor da Internet no Brasil (CGI.br), mostra que 3 a cada 4 brasileiros utilizam a internet, porém 20 milhões de domicílios (28\%) não possuem conexão à Internet, realidade que afeta especialmente famílias com renda de até um salário-mínimo (45\%). Isso evidencia uma desigualdade digital presente no país, o que realmente pode comprometer o acesso de uma parcela dos pacientes a serviços de telemedicina. Já os demais entrevistados se posicionaram contrários quanto a existência de restrição tecnológica, conforme o E3 "sistemas de smartphones permitem baixar aplicativos que podem ser usados nesse contexto da telemedicina" e o E1 "Todo mundo praticamente tem acesso à internet e celular".

A quinta questão era sobre a relação médico-paciente em telemedicina. Consoante o E1 "Acho que o médico consegue achar o diagnóstico mais rápido por meio do olhar, da conversa, do contato físico com o paciente" e o E2 "Eu gosto de manter uma confiança com o médico, o afeto que passa para mim numa consulta é muito importante". Essas respostas exprimem o quanto alguns pacientes valorizam a presença e o contato físico com seus médicos para que se sintam confiantes numa consulta. Logo, talvez isto seja um elemento de resistência, afinal, o processo comunicativo permeia todas as relações interpessoais e a forma como é desenvolvido pode facilitar ou dificultar a confiança e o vínculo do paciente e família com os profissionais de saúde, assim como o vínculo destes com o serviço de saúde, influenciando na adesão ao tratamento (Otani \& Aparecida, 2013). Em contrapartida, o E3 considera que a relação médico-paciente não fica tão prejudicada na telemedicina, mas pondera: "Penso que tenha que ser por videoconferência para tornar a consulta algo amigável para o paciente, para se sentir confortável".

O último questionamento era se a telemedicina traria redução de custos para o paciente/hospital/ambos. Para o E3 “Todo mundo sai ganhando. Para o paciente vai poupar tempo, não vai ficar esperando. Para o médico, não vai precisar manter uma grande estrutura para fazer seus atendimentos". Essa opinião do entrevistado está de acordo com as prioridades e objetivos dos serviços por telemedicina que são justamente atender de forma remota os pacientes e com isso reduzir custos operacionais que implicam num menor gasto para o paciente ao utilizar essas formas de atendimento clínico e também para os hospitais/clínicas por não precisar de uma grande estrutura física para atender uma demanda elevada de consultas, por exemplo. Apesar disso, contrário a essa opinião, o E4 afirma: "Pro hospital não vai gastar tanto, economiza. Mas, o paciente vai sempre ter que gastar, não vai trazer redução não".

\section{Conclusão}

O resultado prático encontrado nesta pesquisa revela que maioria dos pacientes, seja do SUS ou convênio/particular, considera que usaria serviços de telemedicina, contudo, os entrevistados ponderaram que priorizam o atendimento presencial, e somente utilizariam essa alternativa tecnológica de teleconsulta para acompanhamento clínico ou se houver inviabilidade da consulta tradicional física. 
Observou-se também que, apesar de a telemedicina ser uma das aplicações médicas mais comuns da atualidade, boa parte dos pacientes entrevistados não a conhecem. Ademais, as respostas apontaram uma compreensão limitada acerca da abrangência e aplicações da telemedicina, haja vista que somente teleconsulta foi citada. Porém, essa é a modalidade com a qual os pacientes irão usar de forma mais frequente, o que justifica esse resultado.

Além disso, os entrevistados apresentaram respostas bem formuladas e diversificadas sobre as vantagens e desvantagens da telemedicina, apesar do foco dessas análises ter sido acerca da teleconsulta. Muitos desses pacientes, não somente expuseram suas opiniões, como também associaram as práticas da telemedicina com sua realidade e opinaram como as utilizariam. Das principais limitações da pesquisa, além daquelas inerentes às características do método escolhido, destaca-se a presença maior da faixa etária adulto jovem dos entrevistados, com poucas pessoas idosas, as quais estão menos habituadas a utilizar tecnologias. Outro fator é quanto ao grau de escolaridade, em que muitos dos entrevistados já tinham alguma formação superior, o que lhes confere mais utilização de internet, celulares; e consequentemente uma maior aceitação da utilização dos serviços de telemedicina. Portanto, como proposta futura para uma nova pesquisa, poderiam ser incluídas pessoas com maior diversidade de idade e de formação, para melhor compreender as demandas e opiniões dos pacientes sobre essa modalidade tecnológica de serviço médico que cada vez mais se amplia.

Assim, os sistemas de saúde, sejam públicos ou privados, possuem o desafio de ofertar um atendimento efetivo aos pacientes por telemedicina. Este atendimento, porém, pelos resultados obtidos neste estudo, deve abranger modalidades de segunda consulta para acompanhamento clínico dos pacientes, uma vez que há predileção pela consulta presencial como forma de primeiro atendimento. Dessa forma, a telemedicina pode ser ainda mais aplicada e, assim, ser utilizada como instrumento de integração entre médicos e pacientes, tema que norteou este estudo.

Futuros estudos podem abordar o tema telemedicina ao entrevistar pacientes em outros níveis de cuidados à saúde, como na atenção primária e secundária à saúde e também englobar no escopo do trabalho os profissionais de saúde e gestores para avaliar quais grupos são mais favoráveis e desfavoráveis a telemedicina.

\section{Referências}

Almino, M. A. F. B., Rodrigues, S. R., Barros, K. S. B., Fonteles, A. S., Alencar, L. B. L., Lima, L. L. de, \& Jorge, M. S. B. (2014). Telemedicina: um instrumento de educação e promoção da saúde pediátrica. Revista Brasileira de Educação Médica, 38(3), 397-402. https://doi.org/10.1590/S0100-55022014000300015

Bezerra, M. C. C. S., Júnior, M. G. A. C., \& Moraes, R. B. (2017). Telemedicina: Uma Revisão Sistemática Sobre O Perfil De Consultas Por Videoconferência. II Congresso Brasileiro de Ciências Da Saúde. http://www.editorarealize.com.br/revistas/conbracis/trabalho s/TRABALHO_EV071_MD1 _SA1_ID5\%0A10_12052017191533.pdf

CFM. (2002). RESOLUÇÃO CFM $n^{o}$ 1.643/2002. http://www.portalmedico.org.br/resolucoes/CFM/2002/1643_2002.pdf

CFM. (2019). Código Ética Médica. https://portal.cfm.org.br/images/PDF/cem2019.pdf

Ciommo, R. D. (2019). Telemedicina: Vantagens e desvantagens. https://www.planodesaude.net/telemedicina-vantagens-desvantagens/

Fernandes, N. M. da S., Bastos, M. G., Oliveira, N. A. C. de, Costa, A. do V., \& Bernardino, H. S. (2015). Telemedicine: Development of a distance care system for pre-dialysis chronic kidney disease patients. Jornal Brasileiro de Nefrologia, 37(3). https://doi.org/10.5935/0101-2800.20150055

Jardim, C. (2019). Telemedicina: O papel do paciente e o papel do médico. https://drauziovarella.uol.com.br/coluna-2/telemedicina-o-papel-do-paciente-e-opapel-domedico-coluna/

Luz, P. L. da. (2019). Telemedicine and the Doctor/Patient Relationship. Arquivos Brasileiros de Cardiologia. https://doi.org/10.5935/abc.20190117

Maldonado, J. M. S. de V., Marques, A. B., \& Cruz, A. (2016). Telemedicine: challenges to dissemination in Brazil. Cadernos de Saúde Pública, 32(suppl 2). https://doi.org/10.1590/0102-311X00155615

Mariani, A. W., \& Pêgo-Fernandes, P. M. (2012). Telemedicine: a technological revolution. Sao Paulo Medical Journal, 130(5), 277-278. https://doi.org/10.1590/S1516-31802012000500001

Ministério da Saúde. (2020). Portaria No 467, de 20 de março de 2020. Diário Oficial Da União, 737(1501771), 1-6. http://www.in.gov.br/en/web/dou/-/portarian-467-de-20-de-marco-de-2020-249312996 
Research, Society and Development, v. 10, n. 9, e41610918496, 2021

(CC BY 4.0) | ISSN 2525-3409 | DOI: http://dx.doi.org/10.33448/rsd-v10i9.18496

Nunes, A. A., Bava, M. do C. G. C., Cardoso, C. L., Mello, L. M. de, Trawitzki, L. V. V., Watanabe, M. G. de C., Braggion, M. F., Matumoto, S., Carreta, R. D., \& Santos, V. dos. (2016). Telemedicina na Estratégia de Saúde da Família: avaliando sua aplicabilidade no contexto do PET Saúde. Cadernos Saúde Coletiva, 24(1), 99-104. https://doi.org/10.1590/1414-462X201600010187

Oliveira, M. R., \& Chao, L. W. (2000). Telemedicina FMUSP.

Otani, M. A. P. (2013). Comunicação Entre Profissional De Saúde E Paciente: percepções de mulheres com câncer de mama.

Pereira A. S. et al. (2018). Metodologia da pesquisa científica. UFSM.

Pereira, C. C. A., \& Machado, C. J. (2015). Telessaúde no Brasil - conceitos e aplicações. Ciência \& Saúde Coletiva, $20(10), 3283-3284$. https://doi.org/10.1590/1413-81232015209.07082015

Schmitz, C. A. A., Rodrigues Gonçalves, M., Nunes Umpierre, R., Da Silva Siqueira, A. C., Pereira D’Ávila, O., Goulart Molina Bastos, C., Dal Moro, R. G., Katz, N., \& Harzheim, E. (2017). Teleconsulta: nova fronteira da interação entre médicos e pacientes. Revista Brasileira de Medicina de Família e Comunidade, 12(39), 1-7. https://doi.org/10.5712/rbmfc12(39)1540

SES/RS. (2014). Serra é a quinta macrorregião gaúcha a implantar telediagnóstico para doenças respiratórias crônicas. https://estado.rs.gov.br/serra-e-aquinta-macrorregiao-gaucha-aimplantar-telediagnostico-para-doencas-respiratorias-cronicas

Silveira, E. (2020). Pandemia aumenta alcance da telemedicina no Brasil. https://www.revistaquestaodeciencia.com.br/index.php/questao-defato/2020/07/17/pandemia-aumenta-alcance-da-telemedicina-no-brasil

Urtiga, K. S., Louzada, L. A., \& Lúcia, C. (2004). Telemedicina: uma visão geral do estado da arte. IX Congresso Brasileiro de Informática Em Saúde.

Wen, C. L. (2008). Telemedicina e Telessaúde - Um panorama no Brasil. Informática Pública, 10(2). 